\title{
Trajectories of Diabetes Medication Adherence and Hospitalization Risk: A Retrospective Cohort Study in a Large State Medicaid Program
}

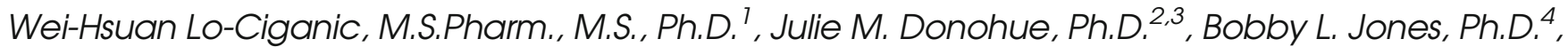 \\ Subashan Perera, Ph.D. ${ }^{5,6}$, Joshua M. Thorpe, Ph.D., M.P.H. ${ }^{3,7,8}$, Carolyn T. Thorpe, Ph.D., M.P.H. ${ }^{3,7,8}$, \\ Zachary A. Marcum, Pharm.D., Ph.D. ${ }^{9}$, and Walid F. Gellad, M.D., M.P.H. ${ }^{3,6,8}$
}

\begin{abstract}
'Department of Pharmacy Practice and Science, College of Pharmacy, University of Arizona, Tucson, AZ, USA; ${ }^{2}$ Department of Health Policy and Management, Graduate School of Public Health, University of Pittsburgh, Pittsburgh, PA, USA; ${ }^{3}$ Center for Pharmaceutical Policy and Prescribing, University of Pittsburgh, Pittsburgh, PA, USA; ${ }^{4}$ Department of Psychiatry, University of Pittsburgh Medical Center, Pittsburgh, PA, USA; ${ }^{5}$ Department of Biostatistics, Graduate School of Public Health, University of Pittsburgh, Pittsburgh, PA, USA; ${ }^{\circ}$ Department of Medicine, School of Medicine, University of Pittsburgh, Pittsburgh, PA, USA; ${ }^{7}$ Department of Pharmacy and Therapeutics, School of Pharmacy, University of Pittsburgh, Pittsburgh, PA, USA; ${ }^{8}$ Center for Health Equity Research and Promotion, Veterans Affairs Pittsburgh Healthcare System, Pittsburgh, PA, USA; ${ }^{9}$ Department of Pharmacy, School of Pharmacy, University of Washington, Seattle, WA, USA.
\end{abstract}

\begin{abstract}
BACKGROUND: Numerous interventions are available to boost medication adherence, but the targeting of these interventions often relies on crude measures of poor adherence. Group-based trajectory models identify individuals with similar longitudinal prescription filling patterns. Identifying distinct adherence trajectories may be more useful for targeting interventions, although the association between adherence trajectories and clinical outcomes is unknown.
\end{abstract}

OBJECTIVE: To examine the association between adherence trajectories for oral hypoglycemics and subsequent hospitalizations among diabetes patients.

DESIGN: Retrospective cohort study.

PATIENTS: A total of 16,256 Pennsylvania Medicaid enrollees, non-dually eligible for Medicare, initiating oral hypoglycemics between 2007 and 2009.

MAIN MEASURES: We used group-based trajectory models to identify trajectories of oral hypoglycemics in the 12 months post-treatment initiation, using monthly proportion of days covered (PDC) as the adherence measure. Multivariable Cox proportional hazard models were used to examine the association between trajectories and time to first diabetes-related hospitalization/emergency department (ED) visits in the following year. We used the $\mathrm{C}$-index to compare prediction performance between adherence trajectories and dichotomous cutpoints (annual PDC $<80$ vs. $\geq 80 \%$ ).

RESULTS: The mean annual PDC was 0.58 (SD 0.32). Seven trajectories were identified: perfect adherers $(9 \%$ of the cohort), nearly perfect adherers (31.4\%), moderate adherers $(21.0 \%)$, low adherers (11.0\%), late discontinuers (6.8\%), early discontinuers (9.7\%), and non-adherers with only one fill (11.1\%). Compared to perfect adherers, trajectories of moderate adherers ( $\mathrm{HR}=1.48,95 \% \mathrm{CI} 1.25,1.75)$, low adherers ( $\mathrm{HR}=1.51,95 \%$ CI 1.25, 1.83), and non-adherers with only one fill (HR $=1.35,95 \%$ CI $1.09,1.67)$ had greater

Electronic supplementary material The online version of this article (doi:10.1007/s11606-016-3747-6) contains supplementary material, which is available to authorized users.

Received December 11, 2015

Revised April 18, 2016

Accepted May 4, 2016

Published online May 26, 2016 risk of diabetes-related hospitalizations/ED visits. Predictive accuracy was improved using trajectories compared to dichotomized cutpoints (C-index $=0.714$ vs. 0.652).

CONCLUSIONS: Oral hypoglycemic treatment trajectories were highly variable in this large Medicaid cohort. Low and moderate adherers and those filling only one prescription had a modestly higher risk of hospitalizations/ED visits compared to perfect adherers. Trajectory models may be valuable in identifying specific non-adherence patterns for targeting interventions.

KEY WORDS: diabetes; oral hypoglycemics; medication adherence; groupbased trajectory models; Medicaid.

J Gen Intern Med 31(9): 1052-60

DOI: $10.1007 / \mathrm{s} 11606-016-3747-6$

(C) Society of General Internal Medicine 2016

\section{INTRODUCTION}

Diabetes affects over 29 million Americans and poses an enormous economic burden, accounting for $\$ 176$ billion in direct costs in 2012. ${ }^{1}$ High diabetes-related costs are due, in part, to the development of complications, hospitalizations, premature disability, and mortality as a consequence of suboptimal medication adherence. ${ }^{2,3}$ Numerous interventions for boosting medication adherence are available for patients with diabetes, but targeting of these interventions often relies on crude measures of poor adherence that assume all patients having identical nonadherence patterns over time. In order to design targeted and tailored interventions to improve adherence, methods are needed to identify distinct non-adherence patterns, since medication use is a dynamic behavior that may change over time. ${ }^{4}$

Adherence is routinely measured based on prescription refills using administrative claims data. These claims-based measures are used in health plan quality ratings, in identifying non-adherent patients for targeted interventions by health 
systems, in research investigating the impact of medication non-adherence on clinical outcomes ${ }^{3,5-9}$ and in predicting health care costs and utilization. ${ }^{10-12}$ However, most studies, and all current quality reporting measures, use a single adherence measure to represent average medication refills in a year (e.g., proportion of days covered [PDC] above a predefined threshold). The use of a single measure of adherence may mask substantial underlying heterogeneity in refill patterns that could have important implications for intervention effectiveness and, ultimately, patient outcomes and healthcare costs. For example, an average annual adherence measure could assign the same level of adherence to a patient who was partially adherent for 1 year and a patient who was fully adherent for 6 months and then discontinued treatment.

Group-based trajectory models identify patient subgroups with similar patterns of medication refills and summarize the trajectory of average adherence for each group over time in an easily understandable graphical depiction. ${ }^{13-16}$ In prior work, trajectory models have been applied to statin adherence, demonstrating variability in adherence patterns over time and an association with cardiovascular events. ${ }^{17}$ However, no studies have examined the association between adherence trajectories for oral hypoglycemics and clinical outcomes in diabetes, or compared their predictive performance to traditional adherence metrics. In this study, we used group-based trajectory models to identify distinct patterns of oral hypoglycemic refills over time in a large state Medicaid program. ${ }^{13-16}$ We examined the association between these trajectories, as opposed to traditional dichotomous adherence measures (i.e., annual PDC $\geq 80$ vs. $<80 \%$ ), and subsequent diabetes-related and all-cause hospitalizations or emergency department (ED) visits. Finally, we compared prediction performance using adherence trajectories versus dichotomous measures.

\section{METHODS}

\section{Data Sources}

This study was a retrospective cohort analysis of Pennsylvania Medicaid administrative claims data for all fee-for-service and managed care enrollees from 2007 to 2011. Enrollment exceeded 2 million annually. The datasets include enrollment files, Medicaid claims, and encounter data for outpatient, inpatient, long-term care, professional services, and prescription drug claims. Prescription data contain national drug codes, date of prescription fill, quantity dispensed, days of supply, and prescriber information. This study was deemed exempt human subjects research by the University of Pittsburgh Institutional Review Board.

\section{Study Design and Cohort}

We limited our analyses to enrollees aged 18-64 years who were not dually eligible for Medicare, because we were unable to observe Part D prescriptions. Type 2 diabetes patients were identified based on the presence of any International Classification of Diseases, Ninth Revision, Clinical Modification (ICD-9) codes 250.0x through 250.9x (where $\mathrm{x}=0$ or 2) on outpatient, inpatient, or professional claims, and/or having any oral hypoglycemic claims. ${ }^{18}$ An index date was defined as the date of a patient's first prescription fill for oral hypoglycemics between July 1, 2007, and December 31, 2009, with no antidiabetes prescriptions in the prior 6 months (i.e., new users). We followed each patient's refill claims for 12 months following the index date. Patients were required to have $\geq 18$ months of continuous enrollment (i.e., 6 months pre-index date as baseline period and 1 year post) to obtain information on predictors and allow for complete follow-up for adherence measurement. We excluded patients who had 1) a diagnosis of gestational diabetes, 2) metformin used for polycystic ovary syndrome only, 3) insulin, pramlintide, or exenatide prescriptions only, or 4) prolonged institutionalization (i.e., cumulative nursing home or inpatient stay $\geq 90$ days) during the index year, because medications were likely administered by nurses or caregivers (Fig. 1).

\section{Outcome Measures}

Our primary outcome variable was time to first diabetesrelated hospitalizations/ED visit during the 12 months after the first year of treatment. Diabetes-related hospitalizations included inpatient admissions for diabetes, hyperglycemia, hypoglycemia, electrolyte imbalance, diabetes retinopathy, nephropathy and neuropathy, or peripheral circulatory disorder, and were identified by a qualifying ICD-9 code as the primary discharge diagnosis or current procedural terminology (CPT) codes in any position (see online appendix, eTable 1). ${ }^{19-25}$ Our secondary outcome was time to first allcause hospitalization/ED visit during the 12 months after the first year of treatment, given that medication non-adherence may manifest as numerous different adverse outcomes (e.g., falls) and, therefore, increase the risk of all-cause hospitalizations/ED visits. ${ }^{3,26-29}$

\section{Exposures: Adherence to Oral Hypoglycemics}

We constructed two adherence measures in the first year of oral hypoglycemic treatment: 1) a traditional measure using average annual PDC, ${ }^{30-32}$, and 2) the trajectory of adherence defined by monthly PDC with oral hypoglycemics over the first year. Group-based trajectory models account for both the timing and extent of non-adherence and can therefore identify more heterogeneity in non-adherence behavior than traditional single measures.

Adherence Measure. Based on dispensing date and days supplied, we created a supply diary for each patient-day by stringing together consecutive fills of oral hypoglycemics. ${ }^{33}$ First, we calculated an interval-based PDC with oral hypoglycemics over a 1-year period starting from the index oral hypoglycemic prescription. ${ }^{20,30}$ For those with concurrent prescriptions of multiple oral hypoglycemic classes, we 


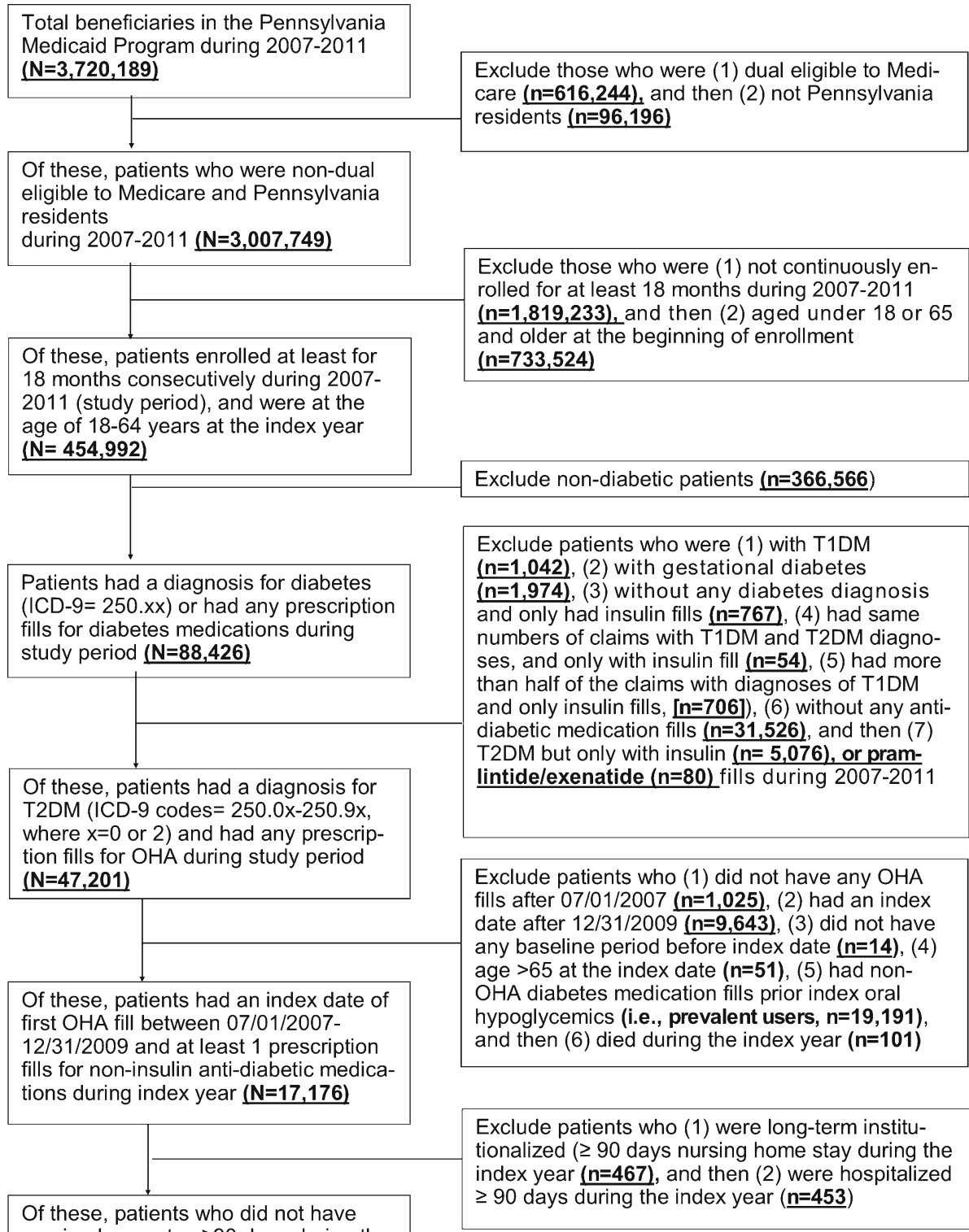

Figure 1 Sample Size Flow Chart. Abbreviations: OHA: oral hyperglycemic agents; T1DM: type 1 diabetes mellitus; T2DM: type 2 diabetes mellitus. Note: We included two other exclusion criteria but they were not listed in the chart because $n=0$ : (1) women who used metformin only, had a diagnosis for polycystic ovary syndrome, but no diagnosis for diabetes; (2) hyperglycemia not otherwise specified (ICD-9 790.6 without any diabetes code).

calculated an average of the PDC across oral hypoglycemic classes and then generated a group mean of these averages over a 1 -year period. ${ }^{30}$ Second, we calculated monthly PDC with $\geq 1$ oral hypoglycemic medication (i.e., the number of days covered with oral hypoglycemic/30 days), given that $95 \%$ of oral hypoglycemic claims were 30-day prescriptions. ${ }^{30}$ We considered all oral hypoglycemic medications as interchangeable when calculating monthly PDC. ${ }^{30}$ When a dispensing occurred before the previous dispensing should have run out, utilization of the new oral hypoglycemic fill was assumed to begin the day after the end of the previous dispensing. We adjusted PDC for inpatient stays, as recommended by Medicare. ${ }^{20}$
Group-Based Trajectory Models. Group-based trajectory models were used to identify and characterize patient subgroups following differential trajectories of adherence over time. ${ }^{34,35}$ We first transformed the monthly PDC with oral hypoglycemics using the arcsine transformation, and modeled the monthly PDC using a censored normal distribution; the time variable was months since initiating treatment (1-12). The purpose of the data transformation was to meet the assumption for the finite mixture trajectory model with a censored normal distribution for each trajectory. ${ }^{34,35}$ We used the most flexible functional form of time, using up to a fifth-order polynomial to allow the trajectories to emerge from the data. The output of trajectory models 
includes estimated group probabilities and trajectory curves of PDC. ${ }^{36,37}$ The final model was selected based on 1) Bayesian information criterion (BIC), wherein the largest value indicates the best-fitting model, and 2) application of Nagin's criteria to assess final model adequacy. ${ }^{36}$

\section{Covariates}

Our covariates, including sociodemographic characteristics, measures of service use, health status, and diabetes treatment/care-related factors, were based on prior studies of adherence. ${ }^{10,38-46}$ We focused on factors measured on or before initiation of oral hypoglycemics in order to avoid including as predictors changes in patient health status that may themselves be consequences of the use (or non-use) of oral hypoglycemics. Sociodemographic factors included age, gender, race/ethnicity (white, black, Hispanic, or other), type of health plan (fee-for-service or managed care plan), and Medicaid eligibility category (General Assistance, Supplemental Security Income, Temporary Assistance for Needy Families [TANF], or Waiver). Measures of service use (measured in the 6 months before the index prescription) included numbers of outpatient visits, ED visits, and hospitalizations, the number of prescribers, average monthly number of prescriptions, and number of pharmacies. ${ }^{43}$ Health status factors included patient comorbidity, defined by the Elixhauser comorbidity index (excluding diabetes, range: $0-29)^{47,48}$, and diabetes severity as defined by the diabetes complications severity index (DCSI, range 0-13) during the baseline period. ${ }^{49,50}$ Finally, we adjusted for diabetes treatment/carerelated factors in the year after treatment initiation (i.e., index year). We included the number of anti-diabetic medication classes and duration of insulin therapy during the index year. We also included variables for having $\mathrm{HbA1C}$ tests, eye exams, nephropathy screenings, or LDL-C tests based on the procedure codes to adjust for general adherence behaviors (see online appendix, eTable 2). ${ }^{51-53}$

\section{Statistical Analysis}

We compared characteristics across identified trajectories with appropriate statistical tests (e.g., chi-square tests). We used multivariable Cox proportional hazards models to assess the association between oral hypoglycemic trajectories and time to first hospitalization/ED visit within the 12 months after the first-year treatment. Enrollees who discontinued Medicaid or died were censored events. Statistical significance was determined using $p<0.05$.

To ensure robustness of the results, we conducted two additional analyses. First, given that medication adherence may have more a direct influence on short-term outcomes, we examined the association between trajectories and risk of outcomes during the 3 months instead of 12 months after the first year of treatment. The results from this analysis were qualitatively similar to our primary analysis; therefore, we present only the main results. Second, we conducted a post hoc analysis comparing the prediction performance of the models using adherence trajectories versus dichotomized cutpoints (PDC $<80 \%$ vs. $\geq 80 \%$ ). We used the Harrell concordance index (C-index) to assess prediction performance. ${ }^{54-56}$ The $\mathrm{C}$-index measures the ability of a model to discriminate between patients who did and did not have events (range 0.5 [completely non-predictive] to 1 [perfectly predictive]). All analyses were performed using Stata ${ }^{\circledR} 13$ (StataCorp LP, College Station, TX) and the TRAJ procedure for trajectory models (www.andrew.cmu.edu/user/bjones). ${ }^{13,14,34}$

\section{RESULTS}

\section{Study Cohort Characteristics}

Among 16,256 patients initiating oral hypoglycemics (mean age 45.5 years; $68 \%$ women; Table 1 ), the average number of monthly prescriptions in the 6 months prior to treatment initiation was 4.8 . Overall, $14.6 \%$ had $\geq 1$ insulin prescription in the first year of treatment. The mean annual PDC was 0.58 (SD 0.32), and $36.4 \%$ had PDC $\geq 80 \%$ in the 12 months after treatment initiation.

\section{Adherence Trajectories}

The overall PDC declined from $0.98(0.10)$ in the first month to $0.51(0.43)$ in the 12th month (see online appendix, eFigure 1). In the trajectory analysis, a seven-group model had the best test characteristics based on BIC (-130792.41) and Nagin's criteria (average posterior probability $\geq 0.7$, narrow CIs for estimated probability, and values of the odds correct classification $>5$ for all groups). In Figure 2, these seven trajectories are characterized and labeled according to the timing of discontinuation of treatment and the level of PDC (perfect: $100 \%$, nearly perfect: $80-100 \%$, moderate: $50-<80 \%$, low: $<50 \%$ ). Only $9.0 \%$ of the cohort refilled oral hypoglycemics persistently over the entire year (perfect adherers), and 31.4\% were nearly perfect adherers. Two trajectories refilled oral hypoglycemics consistently at two different PDC levels over time: moderate $(21.0 \%)$ and low $(11.0 \%)$ adherers. Two trajectories were characterized by the timing of discontinuation of treatment: $9.7 \%$ discontinued before 6 months (early discontinuers) and $6.8 \%$ had moderate adherence before 8 months and then discontinued treatment later during the year (late discontinuers). One-tenth $(11.1 \%)$ were non-adherers who refilled only their first oral hypoglycemic prescription.

Table 1 compares patients by adherence trajectory group. Compared to perfect adherers, enrollees who discontinued oral hypoglycemics were younger (39.4- 48.8 vs. 49.7 years), nonwhite (43.3-57.3\% vs. $37.3 \%$ ), and eligible for Medicaid through TANF (15.4-39.1\% vs. $12.3 \%$ ). The groups discontinuing or poorly adhering to oral hypoglycemics had more frequent ED visits (1.0-1.7 vs. 0.9) and higher use of multiple pharmacies (34.7-45.6\% vs. $30.0 \%$ ) at baseline. Patients who discontinued or were poor adherers used fewer anti-diabetes 


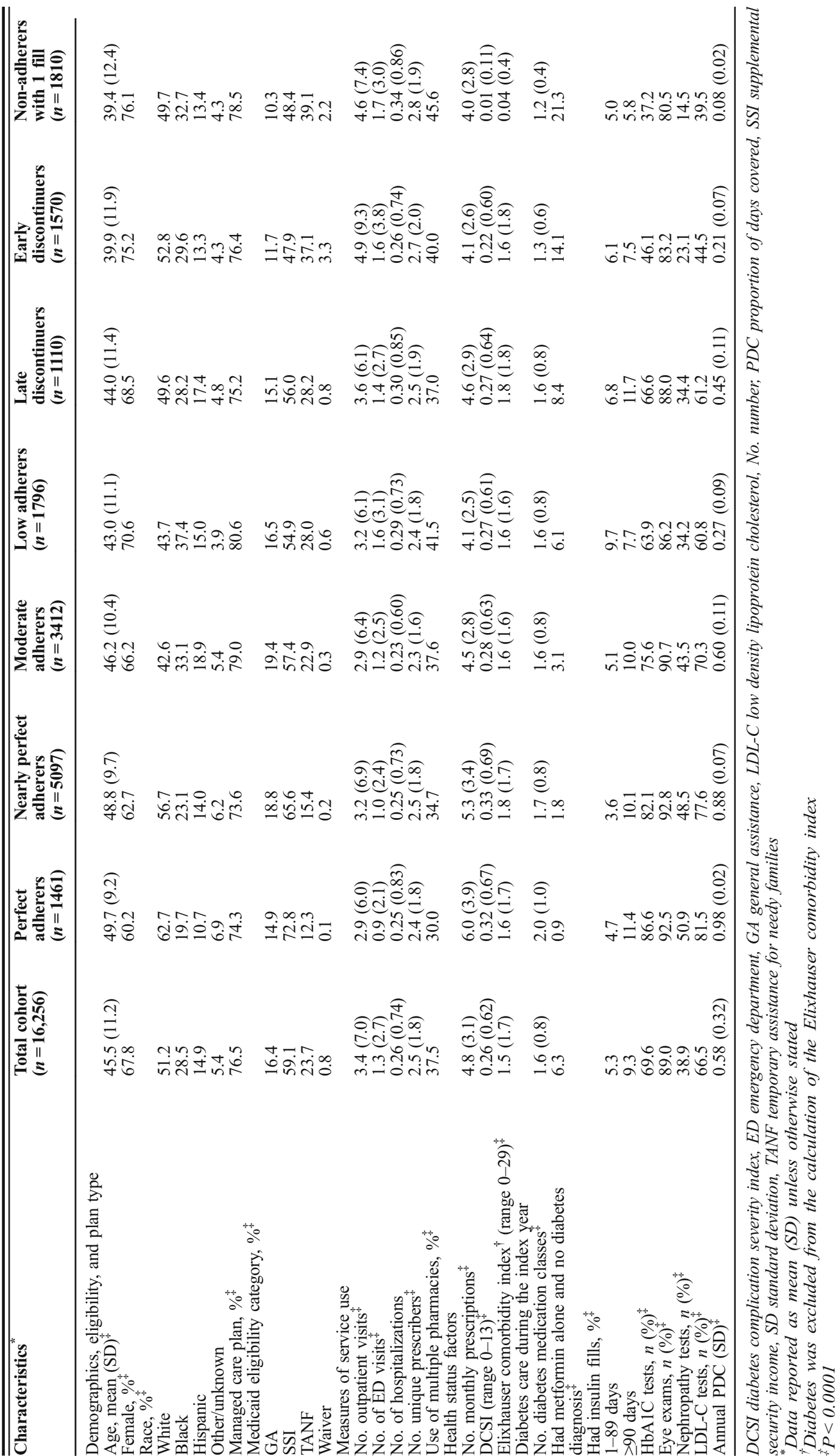




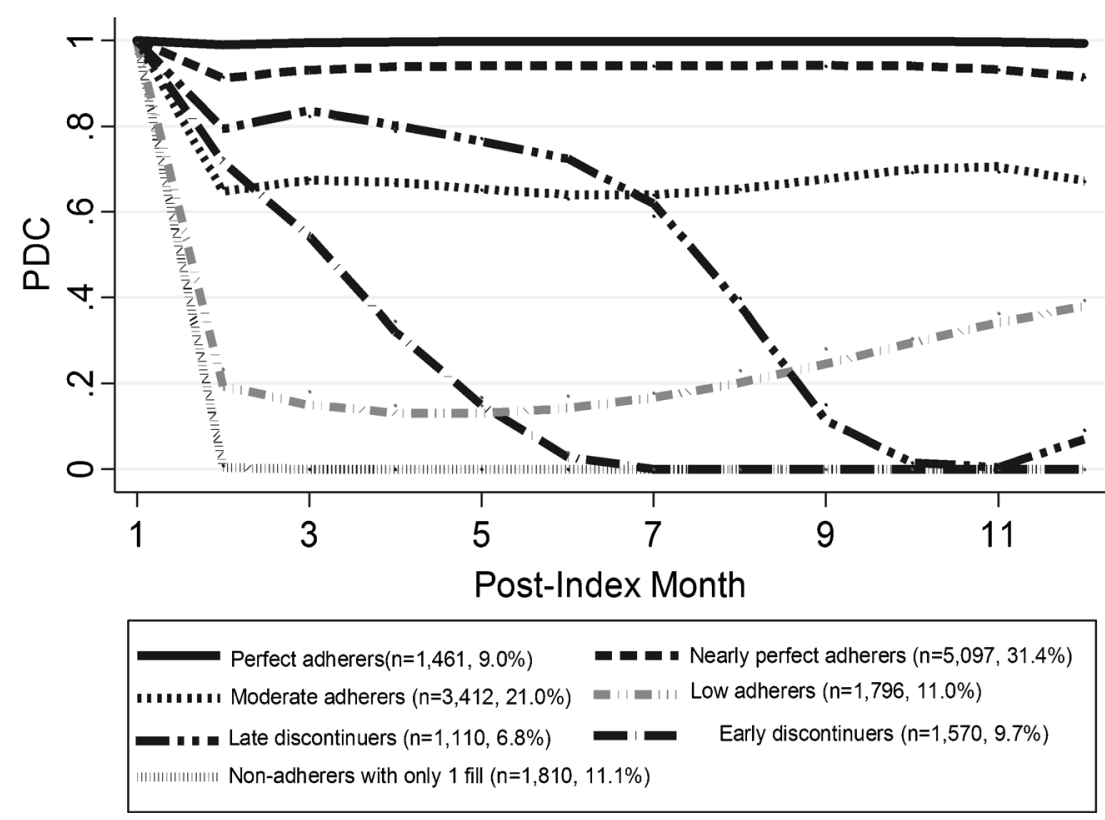

Figure 2 Trajectories of First-Year Adherence to Oral Hypoglycemics. The numbers in parentheses in the legend represent proportions of each trajectory group among all patients.

medication classes $(1.2-1.7$ vs. 2.0$)$ and had fewer diabetesrelated tests (HbA1C tests: $37.2-82.1 \%$ vs. $86.6 \%$; nephropathy tests: $14.5-48.5 \%$ vs. $50.9 \%$; LDL-C tests: $51.4-75.7 \%$ vs. $79.7 \%$ ). These differences were all statistically significant $(p<0.001)$.

\section{Hospitalizations and ED Visits}

Overall, $15.9 \%$ of the sample had diabetes-related hospitalizations/ED visits (median months to event $=10.6$ ), and $55.7 \%$ had all-cause hospitalizations/ED visits (median months to event $=3.5$ ). Compared to perfect adherers, patients in the trajectories of moderate adherers (hazard ratio [HR] = $1.48,95 \%$ CI $1.25,1.75)$, low adherers $(\mathrm{HR}=1.51,95 \% \mathrm{CI}$ $1.25,1.83$ ), and non-adherers with only one oral hypoglycemic fill $(\mathrm{HR}=1.35,95 \%$ CI 1.09, 1.67) had a greater risk of diabetes-related hospitalizations/ED visits in the subsequent year (Table 2). Other factors associated with an increased risk of diabetes-related hospitalizations/ED visits in the following year included higher numbers of ED visits ( $\mathrm{HR}=1.04,95 \%$ CI $1.03,1.05)$, hospitalizations ( $\mathrm{HR}=1.36,95 \%$ CI 1.19 , $1.54)$, and total monthly prescriptions $(\mathrm{HR}=1.03,95 \% \mathrm{CI}$ $1.02,1.05$ ) in the 6 months prior to treatment initiation (see online appendix, eTable 3). Patients who received multiple classes of anti-diabetes medications ( $\mathrm{HR}=1.16,95 \%$ CI 1.08 , 1.23) and had insulin fills $\geq 90$ days ( $\mathrm{HR}=1.29,95 \%$ CI 1.11 , 1.50) during the index year also had an increased risk of diabetes-related hospitalizations/ED visits (see online appendix, eTable 3).

The performance for predicting diabetes-related hospitalizations/ED visits was improved in the multivariate Cox proportional models with trajectory groups $(\mathrm{C}$-index $=$ $0.714,95 \%$ CI $0.682,0.745)$ compared to dichotomized groups $(\mathrm{C}$-index $=0.652,95 \%$ CI $0.614,0.694)$. Similar associations were found for all-cause hospitalizations/ED visits (Table 2) and for diabetes-related and all-cause hospitalizations (see online appendix, eTable 4).

\section{DISCUSSION}

We identified seven adherence trajectories over the first year of oral hypoglycemic treatment. This high variability in treatment underscores the need to understand dynamic patterns of adherence that may provide important information for targeting interventions, over and above cross-sectional measures of adherence (e.g., average PDC). Our study yielded three additional important findings. First, adherence to oral hypoglycemics remains dramatically low. Almost $60 \%$ of new users of oral hypoglycemics discontinued medication at some point in the first year, with most patterns showing a decrease over time or consistent poor adherence. Second, not all of the nonadherence trajectories were associated with significant risk of diabetes-related hospitalizations/ED visits. Patients maintaining low (PDC $\sim 20-40 \%$ ) or moderate adherence (PDC $\sim 60 \%$ ) over the first year and patients filling only one prescription had a $35-51 \%$ greater risk of diabetes-related hospitalizations/ED visits than perfect adherers. Third, including adherence trajectories in the multivariable model showed meaningful improvement in prediction $(>0.025)$ for diabetesrelated hospitalizations/ED visits compared to using the traditional $80 \%$ threshold. $^{56}$

Group-based trajectory models are novel methods that are increasingly applied for examining dynamic adherence patterns. ${ }^{13-16}$ To our knowledge, our study provides some of the first data supporting the predictive validity of adherence 
Table 2 Multivariable Cox Proportional Hazard Models of Adherence Trajectories of Oral Hypoglycemics and Risk of Hospitalizations/ Emergency Department (ED) Visits

\begin{tabular}{|c|c|c|c|c|c|c|c|}
\hline $\begin{array}{l}\text { Trajectory } \\
\text { group }\end{array}$ & $\begin{array}{l}\text { No. in } \\
\text { each } \\
\text { group }\end{array}$ & \multicolumn{3}{|c|}{ Diabetes-related hospitalizations/ED visits } & \multicolumn{3}{|c|}{ All-cause hospitalizations/ED visits } \\
\hline Perfect adherers & 1461 & 14.4 & Referent & - & 46.3 & Referent & - \\
\hline $\begin{array}{l}\text { Nearly perfect } \\
\text { adherers }\end{array}$ & 5097 & 14.4 & $1.06(0.90,1.25)$ & 0.48 & 51.1 & $1.17(1.07,1.28)$ & $<0.0001$ \\
\hline Moderate adherers & 3412 & 18.9 & $1.48(1.25,1.75)$ & $<0.0001$ & 59.5 & $1.47(1.34,1.62)$ & $<0.0001$ \\
\hline $\begin{array}{l}\text { Early } \\
\text { discontinuers }\end{array}$ & 1570 & 13.6 & $1.10(0.89,1.36)$ & 0.36 & 55.4 & $1.31(1.17,1.46)$ & $<0.0001$ \\
\hline $\begin{array}{l}\text { Non-adherers with } \\
1 \text { fill }\end{array}$ & 1810 & 14.0 & $1.35(1.09,1.67)$ & 0.006 & 59.9 & $1.60(1.43,1.79)$ & $<0.0001$ \\
\hline $\begin{array}{l}\text { C-index }(95 \% \text { CI }) \\
\text { PDC } \geq 80 \%\end{array}$ & 5912 & 13.9 & $\begin{array}{l}0.714(0.682,0.745) \\
\text { Referent }\end{array}$ & - & 49.4 & $\begin{array}{l}0.781(0.729,0.828) \\
\text { Referent }\end{array}$ & - \\
\hline $\begin{array}{l}\text { PDC }<80 \% \\
\text { C-index }(95 \% \mathrm{CI})\end{array}$ & 10,344 & 17.0 & $\begin{array}{l}1.38(1.25,1.51) \\
0.652(0.614,0.694)\end{array}$ & $<0.0001$ & 59.3 & $\begin{array}{l}1.29(1.23,1.36) \\
0.769 \\
(0.751,0.786)\end{array}$ & $<0.0001$ \\
\hline
\end{tabular}

Separate multivariable Cox proportional hazard models were used to adjust for sociodemographic factors, health status, and diabetes treatment- and care-related covariates. Final models included age, gender, racelethnicity, type of health plan, type of Medicaid eligibility, number of outpatient visits, number of emergency department visits, number of hospitalizations, number of prescribers, number of unique pharmacies, average number of monthly prescriptions, Elixhauser comorbidity index, and diabetes complication severity index during the 6 months prior or as of the index date. We also included the number of anti-diabetes medication classes, insulin use, and having diabetes-related tests in the 12 months after the index oral hypoglycemic prescription

ED emergency department, HR hazard ratio, PDC proportion of days covered

trajectories with regard to clinical outcomes in diabetes. Consistent with the findings from Franklin and colleagues, ${ }^{15,19}$ our study showed that longitudinal non-adherence patterns were highly variable, with improved prediction performance for clinical outcomes. One of the challenges in applying trajectory models to adherence is measuring concurrent adherence to multiple related medications (e.g., metformin, glyburide); we are aware of no prior trajectory analysis that has overcome this challenge. Given this limitation in trajectory analysis, we assumed all hypoglycemics to be interchangeable in our analysis, which may overestimate adherence. ${ }^{30}$ Methods are needed for identifying simultaneous adherence trajectories in complex treatment regimens.

In light of better predictive performance related to clinical outcomes and an ability to capture changes in adherence, trajectory models may be preferable to traditional crosssectional measures when designing and evaluating adherence interventions. For example, it may be possible to observe nonadherence patterns over a shorter period of time (e.g., 6 months) and customize interventions based on trajectory membership. Using single-value measures of adherence obfuscates distinct non-adherence patterns over time. ${ }^{17}$ These behavioral patterns may reflect levels of engagement in treatment, side effects, or self-management style over time that require different intervention content, timing, duration, and intensity in order to be maximally effective ${ }^{57}$ Using trajectory models in concert with more frequent measurement of adherence (e.g., monthly, quarterly) may help payers and clinicians identify the appropriate timing of interventions to improve adherence, better allocate resources to those with higher risk, and ultimately improve disease management and outcomes.
Not all of the trajectories had a greater risk of hospitalizations/ED visits compared to perfect adherers. For example, this risk among early discontinuers, whose adherence steadily dropped off during the first 6 months, was similar to that of perfect adherers. Early discontinuers appeared to have less severe diabetes and receive fewer diabetes-related tests (e.g., lipid levels), raising the question of whether these individuals have general non-adherence behaviors, or whether perhaps their diabetes improved to the point that medications were no longer needed. Regardless, the differential risk across trajectories, and the improved prediction of hospitalizations/ED visits when using trajectory groups, highlights the potential for developing more accurate predictions about who will need interventions at a given time to prevent adverse outcomes, allowing for the efficient use of scarce resources.

Several limitations should be considered when interpreting these results. First, our study relied on administrative data that lack clinical and socio-behavioral information (e.g., $\mathrm{HbA1C}$ results) and information on reasons for treatment discontinuation (e.g., physician decision). It is unknown whether and how the dispensed drugs were actually used by the patients. Patients who never filled their first prescription (i.e., primary non-adherence) or who had other sources of prescription coverage (e.g., safety net programs, free samples) were also not captured in this analysis. ${ }^{44}$ Second, we required 1-year continuous enrollment to allow for complete follow-up for adherence measures. If patients with intermittent enrollment in Medicaid programs were more likely to be poor adherers, we would underestimate the risk of clinical outcomes among those in non-adherence trajectories. Third, we cannot rule 
out unmeasured confounders (e.g., healthy user bias) in this observational study. ${ }^{58}$ However, including variables for receiving recommended screenings (proxy measures of healthy behavior) did not impact the results. Finally, we cannot rule out the possibility that our sample includes pre-diabetes patients receiving metformin alone or patients who had been non-adherers and were reinitiating treatment. However, the trajectory results and associated risk of hospitalizations/ED visits were similar in two sensitivity analyses: 1) excluding 1022 patients who had metformin alone and no diabetes diagnosis from our cohort; 2) applying a 12-month baseline period to identify new users of oral hypoglycemics. ${ }^{59}$

\section{CONCLUSIONS}

In this large Medicaid cohort, we identified seven distinct trajectories of oral hypoglycemic treatment, with variable association with diabetes-related hospitalizations/ED visits. The use of adherence trajectories instead of cross-sectional measures improves the ability to predict diabetes-related hospitalizations/ED visits from claims data. These trajectories also demonstrate intuitive longitudinal changes in adherence patterns over time that can be used to target and tailor interventions. Such interventions are desperately needed to improve health outcomes in diabetes.

Author Contributions: Drs. Lo-Ciganic, Donohue, and Gellad had full access to all of the data in the study and take responsibility for the integrity of the data and the accuracy of the data analysis. Study concept and design: Lo-Ciganic, Donohue, Jones, J. Thorpe, C. Thorpe, Perera, Marcum, and Gellad. Acquisition of data: Donohue and Gellad. Analysis and interpretation of data: Lo-Ciganic, Jones, and Gellad. Drafting of the manuscript: Lo-Ciganic and Gellad. Critical revision of the manuscript for important intellectual content: Lo-Ciganic, Donohue, Jones, J. Thorpe, C. Thorpe, Perera, Marcum, and Gellad. Statistical analysis: Lo-Ciganic, Jones, and Perera. Obtained funding: Donohue and Gellad. Administrative, technical, or material support: Lo-Ciganic, Donohue and Gellad. Study supervision: Gellad.

Corresponding Author: Wei-Hsuan Lo-Ciganic, M.S.Pharm., M.S., Ph.D.; Department of Pharmacy Practice and Science, College of PharmacyUniversity of Arizona, Tucson, AZ, USA (e-mail: lociganic@pharmacy.arizona.edu).

Compliance with Ethical Standards:

Financial Support: Dr. Perera is supported by the Pittsburgh Claude D. Pepper Older Americans Independence Center (NIA P3O AGAG024827).

Prior Presentations: A portion of these results were presented in preliminary form on June 2, 2015, at the Pittsburgh Conference on the Science of Medication Adherence 2015, Pittsburgh, PA.

Conflict of Interest: Dr. Gellad serves as an unpaid member of the scientific advisory board of HealthPrize, a medication adherence com pany, and has previously consulted for IMS Health on the topic of medication adherence. All other authors declare that they do not have a conflict of interest.

\section{REFERENCES}

1. Centers for Disease Control and Prevention. National diabetes statistics report: estimates of diabetes and its burden in the United States. Atlanta: US Department of Health and Human Services; 2014.

2. Hughes DA, Bagust A, Haycox A, Walley T. The impact of non-compliance on the cost-effectiveness of pharmaceuticals: a review of the literature. Health Econ. 2001;10(7):601-615.

3. Ho PM, Rumsfeld JS, Masoudi FA, et al. Effect of medication nonadherence on hospitalization and mortality among patients with diabetes mellitus. Arch Intern Med. 2006;166(17):1836-1841.

4. Cutrona SL, Choudhry NK, Fischer MA, et al. Targeting cardiovascular medication adherence interventions. J Am Pharm Assoc. 2012;52(3):381-397.

5. Steiner JF, Prochazka AV. The assessment of refill compliance using pharmacy records: methods, validity, and applications. J Clin Epidemiol. 1997;50(1): 105-116.

6. Woltmann EM, Valenstein M, Welsh DE, et al. Using pharmacy data on partial adherence to inform clinical care of patients with serious mental illness. Psychiatr Serv. 2007;58(6):864-867.

7. Parris ES, Lawrence DB, Mohn LA, Long LB. Adherence to statin therapy and LDL cholesterol goal attainment by patients with diabetes and dyslipidemia. Diabetes Care. 2005;28(3):595-599.

8. Bramley TJ, Gerbino PP, Nightengale BS, Frech-Tamas F. Relationship of blood pressure control to adherence with antihypertensive monotherapy in 13 managed care organizations. J Manag Care Pharm. 2006;12(3):239-245.

9. Lau DT, Nau DP. Oral antihyperglycemic medication nonadherence and subsequent hospitalization among individuals with type 2 diabetes. Diabetes Care. 2004;27(9):2149-2153.

10. Stroupe KT, Teal EY, Tu W, Weiner M, Murray MD. Association of refill adherence and health care use among adults with hypertension in an urban health care system. Pharmacotherapy. 2006;26(6):779-789.

11. Sokol MC, McGuigan KA, Verbrugge RR, Epstein RS. Impact of medication adherence on hospitalization risk and healthcare cost. Med Care. 2005;43(6):521-530.

12. Gilmer TP, Dolder CR, Lacro JP, et al. Adherence to treatment with antipsychotic medication and health care costs among Medicaid beneficiaries with schizophrenia. Am J Psychiatry. 2004;161(4):692-699.

13. Franklin JM, Shrank WH, Pakes J, et al. Group-based trajectory models: a new approach to classifying and predicting long-term medication adherence. Med Care. 2013;51(9):789-796.

14. Modi AC, Rausch JR, Glauser TA. Patterns of nonadherence to antiepileptic drug therapy in children with newly diagnosed epilepsy. JAMA. 2011;305(16):1669-1676.

15. Modi AC, Cassedy AE, Guittner AL, et al. Trajectories of adherence to airway clearance therapy for patients with cystic fibrosis. J Pediatr Psychol. 2010;35(9):1028-1037.

16. Glass TR, Battegay M, Cavassini M, et al. Longitudinal analysis of patterns and predictors of changes in self-reported adherence to antiretroviral therapy: Swiss HIV Cohort Study. J Acquir Immune Defic Syndr. 2010;54(2):197-203.

17. Franklin JM, Krumme AA, Tong AY, et al. Association between trajectories of statin adherence and subsequent cardiovascular events. Pharmacoepidemiol Drug Saf. 2015.

18. Gellad WF, Donohue JM, Zhao X, et al. Brand-name prescription drug use among Veterans Affairs and Medicare Part D patients with diabetes: a national cohort comparison. Ann Intern Med. 2013;159(2): 105-114.

19. National Committee for Guality Assurance. Comprehensive Diabetes Care. Washington, DC: NCQA; 2012.

20. Centers for Medicare \& Medicaid Services. Physician Quality Reporting System MEasures Specifications Manual for Claims and Registry Reporting of Individual Measures. Baltimore: CMS; 2011.

21. Menzin J, Korn JR, Cohen J, et al. Relationship between glycemic control and diabetes-related hospital costs in patients with type 1 or type 2 diabetes mellitus. J Manag Care Pharm. 2010;16(4):264-275.

22. Wang J, Imai K, Engelgau MM, Geiss LS, Wen C, Zhang P. Secular trends in diabetes-related preventable hospitalizations in the United States, 19982006. Diabetes Care. 2009;32(7):1213-1217.

23. American Diabetes Association. Economic costs of diabetes in the U.S. In 2007. Diabetes Care. 2008;31(3):596-615. 
24. Coleman RL, Stevens RJ, Holman RR. Estimating risk of clinically evident neuropathy in type 2 diabetes: a UKPDS risk equation. Diabet Med. 2006;23(Suppl 2):51.

25. Ginde AA, Blanc PG, Lieberman RM, Camargo CA Jr. Validation of ICD9-CM coding algorithm for improved identification of hypoglycemia visits. BMC Endocr Disord. 2008;8:4.

26. Sheehan DV, Keene MS, Eaddy M, Krulewicz S, Kraus JE, Carpenter DJ. Differences in medication adherence and healthcare resource utilization patterns: older versus newer antidepressant agents in patients with depression and/or anxiety disorders. CNS Drugs. 2008;22(11):963-973.

27. Wu PH, Yang CY, Yao ZL, Lin WZ, Wu LW, Chang CC. Relationship of blood pressure control and hospitalization risk to medication adherence among patients with hypertension in Taiwan. Am J Hypertens. 2010;23(2):155-160.

28. Simoni-Wastila L, Wei YJ, Qian J, et al. Association of chronic obstructive pulmonary disease maintenance medication adherence with all-cause hospitalization and spending in a Medicare population. Am J Geriatr Pharmacother. 2012;10(3):201-210.

29. Lang K, Federico V, Muser E, Menzin J, Menzin J. Rates and predictors of antipsychotic non-adherence and hospitalization in Medicaid and commercially-insured patients with schizophrenia. J Med Econ. 2013;16(8):997-1006.

30. Choudhry NK, Shrank WH, Levin RL, et al. Measuring concurrent adherence to multiple related medications. Am J Manag Care. 2009;15(7):457-464.

31. Hess LM, Raebel MA, Conner DA, Malone DC. Measurement of adherence in pharmacy administrative databases: a proposal for standard definitions and preferred measures. Ann Pharmacother. 2006;40(78):1280-1288.

32. Chu L-H, Kawatkar A, Gu A. A SAS ${ }^{\circledR}$ Macro Program to Calculate Medication Adherence Rate for Single and Multiple Medication Use. In: Western Users of SAS Software 2011 Conference. 2011. http://www.wuss.org/proceedings 11/Papers_Chu_L_74886.pdf. (Accessed on April 11, 2016).

33. Nau DP. Proportion of Days Covered (PDC) as a preferred method of measuring medication adherence. Pharm Qual Alliance. 2012. http://www.pqaalliance. org/images/uploads/files/PQA\%20PDC\%20vs\%20\%20MPR.pdf. (Accessed April 11, 2016).

34. Jones BL, Nagin DS. A note on a Stata Plugin for estimating group-based trajectory models. Soc Meth Res. 2013;42(4):608-613.

35. Nagin DS, Odgers CL. Group-based trajectory modeling in clinical research. Annu Rev Clin Psychol. 2010;6:109-138.

36. Nagin DS. Group-Based Modeling of Development. Cambridge: Harvard University Press; 2005.

37. Twisk J, Hoekstra T. Classifying developmental trajectories over time should be done with great caution: a comparison between methods. J Clin Epidemiol. 2012;65(10):1078-1087.

38. Andersen RM. Revisiting the behavioral model and access to medical care: does it matter? J Health Soc Behav. 1995;36(1): 1-10.

39. World Health Organization. Adherence to Long-term Therapy: Evidence for action. Geneva: World Health Organization; 2003.

40. Bosworth HB, Olsen MK, Oddone EZ. Improving blood pressure control by tailored feedback to patients and clinicians. Am Heart J. 2005; 149(5):795-803.

41. Bosworth HB, Oddone EZ. A model of psychosocial and cultural antecedents of blood pressure control. J Natl Med Assoc. 2002;94(4):236-248.
42. Murray MD, Morrow DG, Weiner M, et al. A conceptual framework to study medication adherence in older adults. Am J Geriatr Pharmacother. 2004;2(1):36-43.

43. Choudhry NK, Fischer MA, Avorn J, et al. The implications of therapeutic complexity on adherence to cardiovascular medications. Arch Intern Med. 2011;171(9):814-822.

44. Fischer MA, Choudhry NK, Brill G, et al. Trouble getting started: predictors of primary medication nonadherence. Am J Med. 2011;124(11):1081 e9-22.

45. Stroupe KT, Teal EY, Weiner M, Gradus-Pizlo I, Brater DC, Murray MD. Health care and medication costs and use among older adults with heart failure. Am J Med. 2004;116(7):443-450.

46. Thorpe CT, Bryson CL, Maciejewski ML, Bosworth HB. Medication acquisition and self-reported adherence in veterans with hypertension. Med Care. 2009;47(4):474-481.

47. Guan H, Sundararajan V, Halfon P, et al. Coding algorithms for defining comorbidities in ICD-9-CM and ICD-10 administrative data. Med Care. 2005;43(11):1130-1139.

48. Elixhauser A, Steiner C, Harris DR, Coffey RM. Comorbidity measures for use with administrative data. Med Care. 1998;36(1):8-27.

49. Young BA, Lin E, Von Korff $\mathbf{M}$, et al. Diabetes complications severity index and risk of mortality, hospitalization, and healthcare utilization. Am J Manag Care. 2008;14(1):15-23.

50. Chang HY, Weiner JP, Richards TM, Bleich SN, Segal JB. Predicting costs with diabetes complications severity index in claims data. Am $\mathrm{J}$ Manag Care. 2012;18(4):213-219.

51. Stuart BC, Dai M, Xu J, Loh FH, Dougherty JS. Does good medication adherence really save payers money? Med Care. 2015;53(6):517-523.

52. Halanych JH, Wang F, Miller DR, et al. Racial/ethnic differences in diabetes care for older veterans: accounting for dual health system use changes conclusions. Med Care. 2006;44(5):439-445.

53. Healthcare Effectiveness Data and Information Set (HEDIS) Measure 2015 Physician Documentation Guidelines and Administrative Codes. https:// www.anthem.com/provider/noapplication/f5/s2/t0/pw_e192450.pdf? refer=ahpprovider. (Accessed on April 11, 2016).

54. Harrell FE Jr, Lee KL, Mark DB. Multivariable prognostic models: issues in developing models, evaluating assumptions and adequacy, and measuring and reducing errors. Stat Med. 1996;15(4):361-387.

55. Pencina MJ, D'Agostino RB. Overall $\mathrm{C}$ as a measure of discrimination in survival analysis: model specific population value and confidence interval estimation. Stat Med. 2004;23(13):2109-2123.

56. Apfel CC, Kranke P, Greim CA, Roewer N. What can be expected from risk scores for predicting postoperative nausea and vomiting? $\mathrm{Br} \mathrm{J}$ Anaesth. 2001;86(6):822-827.

57. Zullig LL, Gellad WF, Moaddeb J, et al. Improving diabetes medication adherence: successful, scalable interventions. Patient Prefer Adher. 2015;9:139-149.

58. Brookhart MA, Patrick AR, Dormuth C, et al. Adherence to lipidlowering therapy and the use of preventive health services: an investigation of the healthy user effect. Am J Epidemiol. 2007;166(3):348-354.

59. Roberts AW, Dusetzina SB, Farley JF. Revisiting the washout period in the incident user study design: why 6-12 months may not be sufficient. J Comp Eff Res. 2015;4:27-35. 